Research Article

\title{
Projected Adaptive Cubic Regularization Algorithm with Derivative-Free Filter Technique for Box Constrained Optimization
}

\author{
Lingyun He $\mathbb{D}^{1,2}$ Peng Wang, ${ }^{3}$ and Detong $\mathrm{Zhu}^{1}$ \\ ${ }^{1}$ Mathematics and Science College, Shanghai Normal University, Shanghai 200234, China \\ ${ }^{2}$ School of Mathematics and Statistics, Henan University of Science and Technology, Luoyang 471023, China \\ ${ }^{3}$ Mathematics and Statistics College, Hainan Normal University, Haikou, Hainan 570203, China
}

Correspondence should be addressed to Lingyun He; hly@haust.edu.cn

Received 9 June 2021; Accepted 13 October 2021; Published 30 October 2021

Academic Editor: Viktor Avrutin

Copyright (c) 2021 Lingyun He et al. This is an open access article distributed under the Creative Commons Attribution License, which permits unrestricted use, distribution, and reproduction in any medium, provided the original work is properly cited.

An adaptive projected affine scaling algorithm of cubic regularization method using a filter technique for solving box constrained optimization without derivatives is put forward in the passage. The affine scaling interior-point cubic model is based on the quadratic probabilistic interpolation approach on the objective function. The new iterations are obtained by the solutions of the projected adaptive cubic regularization algorithm with filter technique. We prove the convergence of the proposed algorithm under some assumptions. Finally, experiments results showed that the presented algorithm is effective in detail.

\section{Introduction}

In this passage, we investigate the following optimization:

$$
\begin{aligned}
& \min f(y) \\
& \text { s.t. } y=\left(y_{1}, y_{2}, \ldots, y_{n}\right) \in R^{n} \\
& l_{i} \leq y_{i} \leq u_{i} \\
& i=1,2, \ldots, n,
\end{aligned}
$$

where $f: R^{n} \longrightarrow R$ is smooth, but its derivative information is unavailable or unreliable. For each $i, l_{i}$ is not greater than $u_{i}$, and there exists some $i_{k}$ such that $l_{k}$ is less than $u_{k}$ strictly. Define the feasible set and the strict interior by $\Omega$, int $(\Omega)$, respectively.

The structure of this passage is as shown below. The relevant information is reviewed and our algorithm for problem (1) is introduced in Section 2. The corresponding analysis are investigated in Sections 3 and 4, respectively. Then, the numerical experiments are listed in details in Section 5 .

\section{Literature Review}

Optimization (1) forms an ordinary but important class in various circumstances. There are lots of researchers using different tools to solve it ([1-4]). In order to complements the algorithm library for solving this kind of optimization, we extent the cubic regularization algorithm (shorted by ARC), which is presented by Cartis et al. in $[5,6]$, with filter technique to solve (1) by projection measure based on probabilistic models.

The numerical results of classical ARC showed excellent performance. In [7], Cartis et al. extended ARC for unconstrained optimizations to finite-difference version. In [8], the authors extended the bound of $[5,6]$ to nonlinear problems with convex constraints. Gould et al., in [9], updated the parameter in the large-scale least-squares nonlinear optimization. Huang and Zhu, in [3], presented an affine scaling ARC algorithm for derivative-free box constrained minimization using backtracking line search technique to obtain the step size. In the framework of ARC, the authors did not use box constraints' information; thus, 
we turn to an affine scaling technique in [10], which usually combine with the methods of trust region [10, 11]. However, for various reasons, there are many examples in computational science and engineering; their (at least some) derivatives were unavailable or unreliable. However, they may still be desirable to get the optimizations. This situation led researchers to look for the way to solve (1). Recently, there are many papers proposing various different techniques for the optimization without derivatives. Algorithms for bound constrained optimization with global convergence results using the trust region derivative-free methods were presented in $[12,13]$. Powell, in $[14,15]$, proposed the NEWUOA algorithm, which employs a quadratic polynomial interpolation for the unconstrained or box constrained optimization with excellent experiment performance. In [16], Conn et al. gave a derivative-free version of the trust region method. The corresponding model was constructed by using the polynomial interpolation techniques. Li and $\mathrm{Zhu}$, in [17], proposed an affine trust region method to solve (1) by using a backtracking relevant condition and a filter technique.

Motivated by these ideas, we will try our best to introduce the ARC method using filter technique to solve (1) without derivatives under certain assumptions in this paper. In absence of derivatives, we use probabilistic polynomials' interpolation to construct models of $f$. The presented algorithm has two merits. The first merit is that to solve the subproblem which is only needed once at every point under some conditions, but the ARC algorithms need to solve repeatedly in $[5,6]$, in which solving a subproblem is computationally more expensive than using line search to get the trial step. The other merit is that we take a backing line search technique to make the new iteration strictly feasible.

In this paper, unless otherwise noted, we write $\|\cdot\|=$ $\|\cdot\|_{2}$ for brevity.

2.1. Notations. There are many constants and notations displayed in the next sections. For convenience, we collect the following:

$\kappa_{g}$ is bound on $\nabla f(y)$

$\kappa_{h}$ is bound on $\nabla^{2} f(y)$

$\kappa_{n g}$ is bound on $\nabla m(y) \triangleq g(y)$

$\kappa_{H}$ is bound on $\nabla^{2} m(y) \triangleq H(y)$

$D$ affines scaling matrix for $f$

$\bar{D}$ affines scaling matrix for $m$

$\kappa_{D}$ is bound on $D, \bar{D}$

$\bar{\beta}_{i}$ are constants related to $m$

$\kappa$ is projected gradient for $f$

$\bar{\kappa}$ is projected gradient for $m$

$\alpha_{k}$ is the step size at $y_{k}$

$\alpha_{k, l}$ is the $l$ th trial step size at $y_{k}$

\section{Development of the Algorithm}

At first, we review the elements of our presented algorithm. Following the notations proposed by Cartis et al., in $[5,6]$, we give the ARC subproblem as follows:

$$
\min \psi_{k}(d)=\nabla f\left(y_{k}\right)^{T} d+\frac{1}{2} d^{T} \nabla^{2} f\left(y_{k}\right) d+\frac{1}{3} \sigma_{k}\|d\|^{3},
$$

where $\sigma_{k}$ is a dynamic positive parameter.

Definition 1. A mapping $P_{C}: R^{n} \longrightarrow C$ is called the projection onto $C$ if

$$
P_{C}(y)=\underset{x \in C}{\arg \min }\|x-y\|,
$$

where $C \neq \Phi$ and $C$ is closed. $P_{C}(y)=\max \{l, \min \{y, u\}\}$ since $\Omega$ in (1) is a box. $\kappa(y)=\left\|y-P_{\Omega}(y-\nabla f(y))\right\|$ is a well-known criticality measure.

Definition 2 (see $[18,19])$. Function $m$ is said fully quadratic of $f$ in $B\left(y_{k}, \delta_{k}\right)$ if, for all $y \in B\left(y_{k}, \delta_{k}\right)$, there exist some positive constants $\kappa_{e f}, \kappa_{e g}, \kappa_{e h}$, such that

$$
\begin{gathered}
|f(y)-m(y)| \leq \kappa_{e f} \delta_{k}^{3}, \\
\|\nabla f(y)-\nabla m(y)\| \leq \kappa_{e g} \delta_{k}^{2}, \\
\left\|\nabla^{2} f(y)-\nabla^{2} m(y)\right\| \leq \kappa_{e h} \delta_{k} .
\end{gathered}
$$

Next, random models $M_{k}$ will be considered; $m_{k}=M_{k}\left(\omega_{k}\right)$ is denoted for their realizations. $X_{k}$ and $\Delta_{k}$ will be used for random quantities, while their realizations are denoted by $x_{k}=X_{k}\left(\omega_{k}\right)$ and $\delta_{k}=\Delta_{k}\left(\omega_{k}\right)$, respectively.

Definition 3 (see $[18,19]$ ). A sequence $\left\{M_{k}\right\}$ is said ( $p$ )-probabilistically fully quadratic for sequence $\left\{B\left(X_{k}\right.\right.$, $\left.\left.\Delta_{k}\right)\right\}$ if $S_{k}=\left\{M_{k}\right.$ is a full quadratic on sequence $\left.\left\{B\left(X_{k}, \Delta_{k}\right)\right\}\right\}$ satisfy $P\left(S_{k} \mid F_{k-1}^{M}\right) \geq p$, in which $F_{k-1}^{M}=\sigma\left(M_{0}, \ldots, M_{k-1}\right)$ is generated by $M_{0}, \ldots, M_{k-1}$. The random models are probabilistically fully quadratic if $p \geq(1 / 2)$.

Now, we expand (2) to the derivative-free version to solve (1):

$$
\begin{aligned}
\min \varphi_{k}(d) & =g_{k}^{T} d+\frac{d^{T} H_{k} d}{2}+\frac{\sigma_{k}\|d\|^{3}}{3} \\
\text { s.t. } & l \leq x_{k}+d \leq u,
\end{aligned}
$$

where $g_{k}=\nabla m\left(y_{k}\right)$ and $H_{k}=\nabla^{2} m\left(y_{k}\right)$. And, $g_{k} \neq \nabla f\left(y_{k}\right)$ and $H_{k} \neq \nabla^{2} f\left(y_{k}\right)$ in the derivative-free case, so $\bar{\kappa}(y) \stackrel{\text { def }}{=}\left\|y-P_{\Omega}(y-\nabla m(y))\right\| \neq \kappa(y)$.

Filter technique was proposed firstly by Fletcher and Leyffer for constrained nonlinear optimization. The idea of using filter is to interpret the system of (1) as a biobjective optimization with two goals: minimizing $f(y)$ and minimizing $\|h(y)\|$, where $h(y)=\bar{D}(y) g(y), \quad \bar{D}(y)=\operatorname{diag}$ $\{\bar{v}(y)\}$, and 


$$
[\bar{v}(y)]_{j}= \begin{cases}1, & \text { if } l_{j}=-\infty \text { and } u_{j}=+\infty \\ \min \left\{y_{j}-l_{j}+\mu \max \left\{0,-[\nabla m(y)]_{j}\right\},\right. & \\ u_{j}-y_{j}+\mu \max \left\{0,[\nabla m(y)]_{j}\right\}, & \text { otherwise. }\end{cases}
$$

Then, we also definite $D(y)$ is the derivative version of $\bar{D}(y)$.

In order to ensure $f$ or $\|h\|$ decreasing sufficiently, we give such a strategy:

$$
\left(1-\eta_{h}\right)\left\|h\left(y_{k}\right)\right\| \geq\left\|h\left(y_{k}\left(\alpha_{k, l}\right)\right)\right\| \text {, }
$$

or

$$
f\left(y_{k}\right)-\eta_{f} \alpha_{k, l}\left\|h\left(y_{k}\right)\right\|^{2} \geq f\left(y_{k}\left(\alpha_{k, l}\right)\right)
$$

where $y_{k}\left(\alpha_{k, l}\right)=y_{k}+\alpha_{k, l} d_{k}$ and $\eta_{h}, \eta_{f} \in(0,1)$ are small positive constants.

If (9) or (10) holds, the current filter $\mathscr{F}$ accepts $y_{k}\left(\alpha_{k, l}\right)$. The filter remains unchanged. Otherwise, neither (9) nor (10) holds; the current filter rejects $y_{k}\left(\alpha_{k, l}\right)$; the filter can be augmented as

$$
\begin{aligned}
\mathscr{F}_{k+1}= & \left\{(\|h\|, f) \in R^{n}:\left(1-\eta_{h}\right)\left\|h\left(y_{k}\right)\right\| \geq\|h\| \text { and } f\left(y_{k}\right)\right. \\
& \left.-\eta_{f} \alpha_{k}\left\|h\left(y_{k}\right)\right\|^{2} \geq f\right\} \cup \mathscr{F}_{k} .
\end{aligned}
$$

At the beginning, initialize $\mathscr{F}_{0}=\left\{(\|h\|, f):\|h\| \geq\|h\|_{0}\right\}$.

Now, we present a projected adaptive cubic regularization algorithm with filter technique (shorted by PFARC) for the solutions of derivative-free box constraint optimization:

3.1. Initialization Step. An initial point $y_{0} \in \operatorname{int}(\Omega)=\{y: l$ $<y<u\}$. Given a positive parameter $\sigma_{0}>0$, a radius $1 \geq \delta_{0}>0$, and constants $\gamma_{1}, \gamma_{2}, \gamma, \lambda_{1}, \lambda_{2}$, they satisfy that

$$
\begin{aligned}
& 0<\gamma_{1} \leq \gamma_{2}<1, \\
& 0<\gamma<1, \\
& 0<\lambda_{1} \leq \lambda_{2}<1 .
\end{aligned}
$$

Initialize the filter $\mathscr{F}_{0}$. Choose $\varepsilon \in(0,1), \eta_{f}, \eta_{h} \in(0,1)$, and $\gamma_{3} \in(0,1)$. Set $k=0$.

\subsection{Main Step}

Step 1: set a interpolation points set $X_{k}$ such that $y_{k} \in X_{k} \subseteq B\left(y_{k}, \delta_{k}\right)$; then, apply Algorithms 6.1 and 6.3 in [20] to construct the fully quadratic model $m_{k}=$ $m\left(y_{k}\right)$ on $B\left(y_{k}, \delta_{k}\right)$. Set

$$
\begin{aligned}
& g_{k}=\nabla m_{k}, \\
& H_{k}=\nabla^{2} m_{k}, \\
& \bar{\kappa}_{k}=\bar{\kappa}\left(y_{k}\right), \\
& h\left(y_{k}\right) \stackrel{\text { def }}{=} \bar{D}_{k} g_{k} .
\end{aligned}
$$

Step 2: if $\bar{\kappa}_{k}=0$, stop at $y_{k}$.

Step 3: solve (7) and obtain $d_{k}$.

Step 4: line search.

Step 4.1: let $l=0$ and $\alpha_{k, 0}=1$.

Step 4.2: set $y_{k}\left(\alpha_{k, l}\right)=y_{k}+\alpha_{k, l} d_{k}$.

Step 4.3: if $\mathscr{F}$ accepts $y_{k}\left(\alpha_{k, l}\right)$, go to Step 4.4, else go to Step 4.6.

Step 4.4: if $\left(1-\eta_{h}\right)\left\|h\left(y_{k}\right)\right\| \geq\left\|h\left(y_{k}\left(\alpha_{k, l}\right)\right)\right\|, y_{k}+\alpha_{k, l}$ $d_{k} \in \Omega$ holds, and accept $\alpha_{k, l}$ and go to Step 4.7, else go to Step 4.5 .

Step 4.5: if $f\left(y_{k}\right)-\eta_{f} \alpha_{k, l}\left\|h\left(y_{k}\right)\right\|^{2} \geq f\left(y_{k}\left(\alpha_{k, l}\right)\right), y_{k}+$ $\alpha_{k, l} d_{k} \in \Omega$ holds, and accept $\alpha_{k, l}$ and go to Step 4.7, else go to Step 4.6.

Step 4.6: set $\alpha_{k, l+1} \in\left[\lambda_{1} \alpha_{k, l}, \lambda_{2} \alpha_{k, l}\right]$; let $l=l+1$; then, go to Step 4.2.

Step 4.7: set $\alpha_{k}=\alpha_{k, l}$ and

$$
s_{k}= \begin{cases}\alpha_{k} d_{k}, & \text { if } y_{k}+\alpha_{k} d_{k} \in \operatorname{int}(\Omega), \\ \theta_{k} \alpha_{k} d_{k}, & \text { if otherwise, }\end{cases}
$$

where $\theta_{k} \in\left(\theta_{0}, 1\right]$, for some $0<\theta_{0}<1$ and $\theta_{k}-1=O\left(\left\|d_{k}\right\|\right)$.

Step 5: let $\widetilde{\delta}_{k}=\delta_{k}$. If $\widetilde{\delta}_{k}>\min \left(\kappa_{k},\left\|s_{k}\right\|\right)$, set $\widetilde{\delta}_{k}=\gamma_{3} \widetilde{\delta}_{k}$, and apply Algorithm 6.1 and 6.3 in [20] to construct fully quadratic model $\widetilde{m}_{k}$ in $B\left(y_{k}, \widetilde{\delta}_{k}\right)$. Set $\widetilde{g}_{k}=\nabla \widetilde{m}_{k}$ and $\widetilde{H}_{k}=\nabla^{2} \widetilde{m}_{k}$. Go to Step 3 to compute $\widetilde{d}_{k}$. Until $\widetilde{\delta}_{k} \leq \min \left(\tilde{\bar{\kappa}}_{k},\left\|\widetilde{\boldsymbol{s}}_{k}\right\|\right)$. Update $m_{k}=\widetilde{m}_{k}, g_{k}=\nabla \widetilde{m}_{k}$, $H_{k}=\nabla^{2} \widetilde{m}_{k}, \bar{\kappa}_{k}=\widetilde{\bar{\kappa}}_{k}$, and $s_{k}=\widetilde{s}_{k}$, and compute $\varphi_{k}\left(s_{k}\right)$.

Step 6: set $y_{k+1}=y_{k}+s_{k}$. Compute $f_{k+1}, m_{k+1}, g_{k+1}$, and $H_{k+1}$.

Step 7: compute ratio $\rho_{k}=\left(f\left(y_{k}\right)-f\left(y_{k+1}\right)\right) /-\varphi_{k}\left(s_{k}\right)$. Set

$$
\sigma_{k+1}= \begin{cases}\gamma^{-1} \sigma_{k}, & \text { if } \rho_{k}<\gamma_{1}, \\ \sigma_{k}, & \text { if } \gamma_{2} \geq \rho_{k} \geq \gamma_{1}, \\ \gamma \sigma_{k}, & \text { if } \rho_{k}>\gamma_{2} .\end{cases}
$$

Step 8: if neither (9) nor (10) holds, augment the filter set, else the filter does not change. 
Step 9: set $x_{0}^{k+1}=y_{k+1}$ and $\delta_{k+1}=\delta_{k}$. Then, the sample set $X_{k+1}=\left\{x_{0}^{k+1}, \ldots, x_{q}^{k+1}\right\}$ is constructed in $B\left(y_{k+1}, \delta_{k+1}\right)$. Compute $g_{k+1}, H_{k+1}$. Set $k=k+1$.

Remark 1. Now, we describe a concrete implementation in Step 3 using a Cauchy step which is constructed by an affine scaled gradient [21]. The search direction $d_{k}$ satisfies $\varphi_{k}\left(d_{k}\right) \leq \varphi_{k}\left(d_{k}^{C}\right)$, where

$$
\begin{aligned}
d_{k}^{C} & =-\zeta_{k}^{C} \bar{D}_{k}^{2} g_{k}, \\
\zeta_{k}^{C} & =\arg \min _{\zeta \in \mathbb{R}_{+}} \varphi_{k}\left(-\zeta \bar{D}_{k}^{2} g_{k}\right), \\
\varphi_{k}(d) & =g_{k}^{T} d+\frac{1}{2} d^{T} H_{k} d+\frac{1}{3} \sigma_{k}\|d\|^{3} .
\end{aligned}
$$

As we all know that the computation of Cauchy condition usually is much cheaper than the computation in Step 3 , we will give a relationship between the critical measure $\bar{\kappa}$ and the affine gradient. Therefore, we can replace the predicted decrease by the Cauchy step at each criticality measure satisfying this relationship.

Remark 2. Similar to Lemma 5.1 in [16], we can prove that Step 5 will terminate in a finite number if $\kappa_{k} \neq 0$.

Remark 3. In Step 4, the scalar $\alpha_{k}$ with $y_{k}+\alpha_{k} d_{k} \in \Omega$ denotes that

$$
\alpha_{k} \stackrel{\text { def }}{=} \min \left\{\max \left\{\frac{u_{j}-\left[y_{k}\right]_{j}}{\left[d_{k}\right]_{j}}, \frac{l_{j}-\left[y_{k}\right]_{j}}{\left[d_{k}\right]_{j}}\right\}, j=1,2, \ldots, n\right\} \text {, }
$$

in which $u_{j}, l_{j},\left[y_{k}\right]_{j}$, and $\left[d_{k}\right]_{j}$ represent the $j$ th elements of $u, l, y_{k}$, and $d_{k}$, respectively.

\section{Global Convergence Analysis}

Next, we will present some properties of the algorithm PFARC which will be referenced in analysis of global and local convergence. Denote the indices set of the augmented filter by $\mathscr{A}$. First, we make some assumptions as following.

Assumption 1. The iterates $y_{k}$ remain in $\mathscr{L}\left(y_{0}\right)$, where $\mathscr{L}\left(y_{0}\right)$ is the level set of $f, \mathscr{L}\left(y_{0}\right)=\left\{y \in R^{n} \mid f\left(y_{0}\right) \geq f(y), y \in \Omega\right\}$.

Assumption 2. Let $f: R^{n} \longrightarrow R$ be twice continuously differentiable and bounded from below, and its gradient is Lipschitz continuous with Lipschitz constants $L_{g}$.
Assumption 3. Assume that $\|D(y)\| \leq \kappa_{D},\|\bar{D}(y)\| \leq \kappa_{D}$, $\|\nabla f(y)\| \leq \kappa_{g}$, and $\left\|\nabla^{2} f(y)\right\| \leq \kappa_{h}$, for all $y \in \mathscr{L}\left(y_{0}\right)$, respectively, where $\kappa_{D}, \kappa_{g}$, and $\kappa_{h}$ are positive constants.

Let $d_{k}$ be a solution to (7); the following equations,

$$
\left(H_{k}+\sigma_{k}\left\|d_{k}\right\| I\right) d_{k}=-g_{k}
$$

are the first-order necessary conditions of (7).

Consequently, from Assumptions 1-3, (5), and the choice of $\delta_{k}$ in Step 5, we can get that

$$
\begin{aligned}
\kappa_{l f} & \leq f(y) \leq \kappa_{u f}, \\
\left\|H_{k}\right\| & \leq \kappa_{h}+\kappa_{e h} \delta_{0} \stackrel{\text { def }}{=} \kappa_{H}, \\
\left\|g_{k}\right\| & \leq\left\|\nabla f\left(y_{k}\right)\right\|+\left\|\nabla f\left(y_{k}\right)-g_{k}\right\| \leq \kappa_{g}+\kappa_{e g} \delta_{0}^{2 \text { def }}=\kappa_{n g} .
\end{aligned}
$$

Let $\bar{\kappa}$ denote any criticality measure for the derivativefree model such that

$$
\bar{\beta}_{1} \bar{\kappa}(y) \leq\|h(y)\| \stackrel{\text { def }}{=}\|\bar{D}(y) \nabla m(y)\|
$$

holds on $\Omega$, for some $\bar{\beta}_{1}>0$. Certainly, $\bar{\beta}_{1} \bar{\kappa}_{k} \leq \kappa_{D} \kappa_{n g}$ and a natural choice for $\bar{\kappa}$ satisfying $(20)$ is $\bar{\kappa}=\|h(y)\| / \beta_{1}$. And, there exist an $\beta_{1}>0$ such that $\beta_{1} \kappa(y)=\|D(y) \nabla f(y)\|$. First, we show that $\|h(y)\|$ is a criticality measure, which can be found in [21].

Lemma 1. The function $\left\|h\left(y_{k}\right)\right\|$ defined in (20) is a critical measure.

Lemma 2. Let $d_{k}$ satisfy the Cauchy decrease condition in Remark 1. If $\bar{\kappa}_{k} \neq 0$, there is a constant $\bar{\beta}_{2}>0$ which only depends on $\bar{\beta}_{1}, \kappa_{D}$, and $\kappa_{H}$ such that the following holds:

$$
-\varphi_{k}\left(d_{k}\right) \geq \bar{\beta}_{2} \bar{\kappa}_{k} \min \left\{1, \sqrt{\frac{\bar{\kappa}_{k}}{\sigma_{k}}}, \bar{\kappa}_{k}\right\} \text {. }
$$

Proof. Set $d_{k}=-\bar{D}_{k}^{2} g_{k}$ and $h\left(y_{k}\right)=\bar{D}_{k} g_{k}$. First, we derive an upper bound to $\varphi_{k}\left(d_{k}^{C}\right)=\varphi_{k}\left(\zeta_{k}^{C} d_{k}\right)=\min \left\{\varphi_{k}\left(\zeta d_{k}\right) ; \zeta \geq\right.$ $\left.0, \zeta d_{k} \in \Omega\right\}$; then, apply $\varphi_{k}\left(d_{k}\right) \leq \varphi_{k}\left(d_{k}^{C}\right)$. It is easy to see that $g_{k}^{T} d_{k}=-\left\|h\left(y_{k}\right)\right\|^{2} \leq-\bar{\beta}_{1}^{2} \bar{\kappa}_{k}^{2}<0$, since, by (20), the maximum step-size $\zeta_{1}$ arrived at the lower bounds to $\Omega-y_{k}$ is

$$
\begin{aligned}
\zeta_{1} & =\min \left\{\frac{\left[y_{k}-l\right]_{j}}{\left|\left[d_{k}\right]_{j}\right|} ;\left[d_{k}\right]_{j} \leq 0\right\} \\
& =\min \left\{\frac{\left[y_{k}-l\right]_{j}}{\left[\bar{D}_{k}\right]_{j j}\left[h\left(y_{k}\right)\right]_{j}} ;\left[g_{k}\right]_{j}>0,\left[y_{k}\right]_{j}>l_{j}\right\} \\
& \geq \min \left\{\frac{\left[y_{k}-l\right]_{j}}{\min \left\{\left[y_{k}-l\right]_{j},\left[u-y_{k}\right]_{j}+\mu\left[g_{k}\right]_{j}\right\}\left\|h\left(y_{k}\right)\right\|} ;\left[g_{k}\right]_{j}>0,\left[y_{k}\right]_{j}>l_{j}\right\} \\
& \geq \frac{1}{\left\|h\left(y_{k}\right)\right\|} .
\end{aligned}
$$


The step-size $\zeta_{2}$ arrived at the upper bounds to $\Omega-y_{k}$ is calculated in the same way:

$$
\zeta_{2}=\min \left\{\frac{\left[u-y_{k}\right]_{j}}{\left[d_{k}\right]_{j}} ;\left[d_{k}\right]_{j} \geq 0\right\} \geq \frac{1}{\left\|h\left(y_{k}\right)\right\|} .
$$

Let $a_{k}=(1 / 3) \sigma_{k}\left\|d_{k}\right\|^{3}, b_{k}=(1 / 2) d_{k}^{T} H_{k} d_{k}$, and $c_{k}=g_{k}^{T}$ $d_{k}$. Noting that $a_{k}>0$ and $c_{k}<0$, the function $\varphi_{k}\left(\zeta d_{k}\right)=$ $\zeta g_{k}^{T} d_{k}+\left(\zeta^{2} / 2\right) d_{k}^{T} H_{k} d_{k}+\left(\zeta^{3} / 3\right) \sigma_{k}\left\|d_{k}\right\|^{3 \text { def }}=a_{k} \zeta^{3}+b_{k} \zeta^{2}+c_{k} \zeta$, $\zeta \geq 0$ attains its global minimum at $\zeta=\zeta_{3}$, where

$$
\begin{aligned}
\zeta_{3} & =\frac{-b_{k}+\sqrt{b_{k}^{2}-3 a_{k} c_{k}}}{3 a_{k}} \\
& =\frac{-c_{k}}{\sqrt{b_{k}^{2}-3 a_{k} c_{k}}+b_{k}} \\
& \geq \frac{1}{\sqrt{(1 / 4) \kappa_{D}^{4} \kappa_{H}+\sigma_{k} \kappa_{D}^{3}\left\|h\left(x_{k}\right)\right\|}+(1 / 2) \kappa_{D}^{2} \kappa_{H}} \\
& \geq \frac{1}{2} \min \left\{\frac{1}{\kappa_{D}^{2} \kappa_{H}}, \frac{1}{\kappa_{D} \sqrt{\kappa_{D} \sigma_{k}\left\|h\left(x_{k}\right)\right\|}}\right\} .
\end{aligned}
$$

We have $\zeta_{k}^{C}=\min \left\{\zeta_{1}, \zeta_{2}, \zeta_{3}\right\}$. If $\zeta_{k}^{C}<\zeta_{3}$, then

$$
\zeta_{k}^{C}\left(\sqrt{b_{k}^{2}-3 a_{k} c_{k}}+b_{k}\right)<-c_{k}
$$

and furthermore,

$$
a_{k}<\frac{c_{k}^{2}+2 b_{k} c_{k} \zeta_{k}^{C}}{-3 c_{k}\left(\zeta_{k}^{C}\right)^{2}}=\frac{-c_{k}-2 b_{k} \zeta_{k}^{C}}{3\left(\zeta_{k}^{C}\right)^{2}}
$$

Then, if $H_{k}$ is positive semidefinite, that is, $b_{k} \geq 0$, we have that

$$
\begin{array}{r}
2 \zeta_{k}^{C} b_{k}<-c_{k}, \\
\zeta_{k}^{C} \sqrt{-3 a_{k} c_{k}}<-c_{k} .
\end{array}
$$

That is,

$$
\begin{aligned}
b_{k}< & -\frac{c_{k}}{2 \zeta_{k}^{C}}, \\
a_{k}< & \frac{c_{k}}{3\left(\zeta_{k}^{C}\right)^{2}}, \\
\varphi_{k}\left(\zeta_{k}^{C} d_{k}\right)= & a_{k}\left(\zeta_{k}^{C}\right)^{3}+b_{k}\left(\zeta_{k}^{C}\right)^{2}+c_{k} \zeta_{k}^{C}<\frac{c_{k}}{3\left(\zeta_{k}^{C}\right)^{2}}\left(\zeta_{k}^{C}\right)^{3} \\
& -\frac{c_{k}}{2 \zeta_{k}^{C}}\left(\zeta_{k}\right)^{2}+c_{k} \zeta_{k}^{C}=-\frac{5}{6} \zeta_{k}^{C}\left\|h\left(x_{k}\right)\right\|^{2} .
\end{aligned}
$$

Otherwise, $b_{k}<0$, we can get that $\left(-3 a_{k} c_{k} \zeta_{k}^{C} / 2 \max \right.$ $\left.\left\{\sqrt{-3 a_{k} c_{k}},-2 b_{k}\right\}\right)<-c_{k}$, from $-3 a_{k} c_{k} \zeta_{k}^{C} /\left(\sqrt{b_{k}^{2}-3 a_{k} c_{k}}-\right.$ $\left.b_{k}\right)<-c_{k}$, which is equivalent to (25).
First, assume that $\max \left\{\sqrt{-3 a_{k} c_{k}},-2 b_{k}\right\}=\sqrt{-3 a_{k} c_{k}}$. Therefore, from (25), we have

$$
\zeta_{k}^{C}\left(\sqrt{5 b_{k}^{2}}+b_{k}\right)<-c_{k}
$$

Noting that $-b_{k}<0$, we can also obtain

$$
-b_{k}<\frac{-c_{k}}{(\sqrt{5}-1) \zeta_{k}^{C}} .
$$

By combining $b_{k}<0$, (26), and (30), we can get that

$$
\begin{aligned}
\varphi_{k}\left(\zeta_{k}^{C}\right) & =a_{k}\left(\zeta_{k}^{C}\right)^{3}+b_{k}\left(\zeta_{k}^{C}\right)^{2}+c_{k} \zeta_{k}^{C}<\frac{2}{3} c_{k} \zeta_{k}^{C} \\
& -\frac{2}{3(\sqrt{5}-1)} c_{k} \zeta_{k}^{C}<-\frac{1}{6} \zeta_{k}^{C}\left\|h\left(x_{k}\right)\right\|^{2} .
\end{aligned}
$$

Now, assume that $\max \left\{\sqrt{-3 a_{k} c_{k}},-2 b_{k}\right\}=-2 b_{k}$; then,

$$
\begin{aligned}
b_{k} & <-\frac{c_{k}+3 a_{k}\left(\zeta_{k}^{C}\right)^{2}}{2 \zeta_{k}^{C}}, \\
\varphi_{k}\left(\zeta_{k}^{C} d_{k}\right) & =a_{k}\left(\zeta_{k}^{C}\right)^{3}+b_{k}\left(\zeta_{k}^{C}\right)^{2}+c_{k} \zeta_{k}^{C} \\
& <a_{k}\left(\zeta_{k}^{C}\right)^{3}-\frac{c_{k}+3 a_{k}\left(\zeta_{k}^{C}\right)^{2}}{2 \zeta_{k}^{C}}\left(\zeta_{k}^{C}\right)^{2}+c_{k} \zeta_{k}^{C} \\
& <-\frac{1}{2} \zeta_{k}^{C}\left\|h\left(x_{k}\right)\right\|^{2} .
\end{aligned}
$$

On the contrary, $\zeta_{k}^{C}=\zeta_{3}=\left(-b_{k}+\sqrt{b_{k}^{2}-3 a_{k} c_{k}}\right) / 3 a_{k}$, and we obtain that

$$
\begin{aligned}
& a_{k}\left(\zeta_{k}^{C}\right)^{3}+\frac{2}{3} b_{k}\left(\zeta_{k}^{C}\right)^{2}+\frac{1}{3} c_{k} \zeta_{k}^{C}=0, \\
& \varphi_{k}\left(\zeta_{k}^{C} d_{k}\right)=a_{k}\left(\zeta_{k}^{C}\right)^{3}+b_{k}\left(\zeta_{k}^{C}\right)^{2}+c_{k} \zeta_{k}^{C}=\frac{1}{3} b_{k}\left(\zeta_{k}^{C}\right)^{2}+\frac{2}{3} c_{k} \zeta_{k}^{C} .
\end{aligned}
$$

If $b_{k}<0$,

$$
\varphi_{k}\left(\zeta_{k}^{C} d_{k}\right)<\frac{2}{3} c_{k} \zeta_{k}^{C}=-\frac{2}{3} \zeta_{k}^{C}\left\|h\left(x_{k}\right)\right\|^{2} .
$$

If $b_{k} \geq 0$, we can have that $b_{k}<-\left(c_{k} / \zeta_{k}^{C}\right)$ from $\zeta_{k}^{C}=\zeta_{3}=\left(-b_{k}+\sqrt{b_{k}^{2}-3 a_{k} c_{k}}\right) / 3 a_{k}$, and then,

$$
\varphi_{k}\left(\zeta_{k}^{C} d_{k}\right)=\frac{1}{3} b_{k}\left(\zeta_{k}^{C}\right)^{2}+\frac{2}{3} c_{k} \zeta_{k}^{C}<\frac{1}{3} c_{k} \zeta_{k}^{C}=-\frac{1}{3} \zeta_{k}^{C}\left\|h\left(x_{k}\right)\right\|^{2} .
$$

Then, combining $\varphi_{k}\left(d_{k}\right) \leq \varphi_{k}\left(d_{k}^{C}\right)=\varphi_{k}\left(\zeta_{k}^{C} d_{k}\right)$ and (20) and (22)-(35), $-\varphi_{k}\left(d_{k}\right) \geq \bar{\beta}_{2} \bar{\kappa}_{k}^{2}$ holds, where $\bar{\beta}_{2}>0$ is a constant which only depends on $\bar{\beta}_{1}, \kappa_{D}$, and $\kappa_{H}$. The proof is completed.

The following lemma from [5] gives a useful bound on the step $d_{k}$.

Lemma 3. If $d_{k}$ is a solution in Step 3, then 


$$
\left\|d_{k}\right\| \leq \frac{3}{\sigma_{k}} \max \left\{\kappa_{H}, \sqrt{\frac{\bar{\beta}_{1} \sigma_{k} \bar{\kappa}_{k}}{\kappa_{D}}}\right\}
$$

Assumption 4. Suppose that $\left\|\nabla_{d} \varphi_{k}\left(d_{k}\right)\right\| \leq \kappa_{\theta} \min \left\{1,\left\|d_{k}\right\|\right\}$. $\left\|g_{k}\right\|$, where $\kappa_{\theta} \in(0,1)$.

Lemma 4. Let Assumptions 1-4 hold and $m_{k}$ be fully quadratic. If

$$
\sqrt{\sigma_{k} \bar{\kappa}_{k}} \geq \frac{9 \bar{\beta}_{1}}{\left(1-\gamma_{1}\right) \bar{\beta}_{2} \kappa_{D}} \max \left\{\left(L_{g}+\kappa_{e g} \delta_{0}\right)+\kappa_{H}, 1, \bar{\kappa}_{k}\right\} \stackrel{\text { def }}{=} \kappa_{H B},
$$

then $\rho_{k} \geq \gamma_{1}$ and $\sigma_{k} \geq \sigma_{k+1}$.

Proof. From (37), we will get that $\bar{\kappa}_{k} \neq 0$ because, otherwise, the algorithm would have terminated; then, $\bar{\kappa}_{k}=0$ will conflict with (37). However,

$$
\rho_{k}-\gamma_{1}=\frac{f\left(y_{k}+s_{k}\right)-f\left(y_{k}\right)-\varphi_{k}\left(s_{k}\right)+\left(1-\gamma_{1}\right) \varphi_{k}\left(s_{k}\right)}{\varphi_{k}\left(s_{k}\right)} .
$$

Firstly, using a Taylor expansion, for some $\xi_{k} \in(0,1)$, the first term of the numerator in the fraction above becomes that

$$
\begin{aligned}
f\left(y_{k}+s_{k}\right) & -f\left(y_{k}\right)-\varphi_{k}\left(s_{k}\right) \\
& =f\left(y_{k}\right)+\nabla f\left(y_{k}+\xi_{k} s_{k}\right)^{T} s_{k}-f\left(y_{k}\right)-g_{k}^{T} s_{k}-\frac{1}{2} s_{k}^{T} H_{k} s_{k}-\frac{\sigma_{k}}{3}\left\|s_{k}\right\|^{3} \\
& \leq\left(\left\|\nabla f\left(y_{k}+\xi_{k} s_{k}\right)-\nabla f\left(y_{k}\right)\right\|+\left\|\nabla f\left(y_{k}\right)-g_{k}\right\|\right) \cdot\left\|s_{k}\right\|+\frac{1}{2} \kappa_{H}\left\|s_{k}\right\|^{2} \\
& \leq \theta_{k} \alpha_{k} \max \left\{\left(L_{g}+\kappa_{e g} \delta_{0}\right)+\kappa_{H}, 1, \bar{\kappa}_{k}\right\} \cdot \frac{9 \bar{\beta}_{1} \bar{\kappa}_{k}}{\kappa_{D} \sigma_{k}},
\end{aligned}
$$

where Assumption 2, Lemma 3, and (37) are used.

Next, we consider the second term of the numerator in the fraction:

$$
\begin{gathered}
\left(1-\gamma_{1}\right) \varphi_{k}\left(s_{k}\right) \leq-\left(1-\gamma_{1}\right) \bar{\beta}_{2} \theta_{k} \alpha_{k} \bar{\kappa}_{k} \min \left\{1, \sqrt{\frac{\bar{\kappa}_{k}}{\sigma_{k}}}, \bar{\kappa}_{k}\right\} \\
\stackrel{(37)}{\leq}-\left(1-\gamma_{1}\right) \bar{\beta}_{2} \theta_{k} \alpha_{k} \bar{\kappa}_{k}^{2} \cdot \sqrt{\frac{1}{\sigma_{k} \bar{\kappa}_{k}}}
\end{gathered}
$$

However, the denominator $\varphi_{k}\left(s_{k}\right)<0$ is from Lemma 2 . Thus, following with (37)-(40), we get that

$$
\begin{aligned}
\rho_{k}-\gamma_{1} & \geq \frac{\theta_{k} \alpha_{k} \max \left\{L_{g}+\kappa_{e g} \delta_{0}+\kappa_{H}, 1, \bar{\kappa}_{k}\right\}\left(9 \bar{\beta}_{1} \bar{\kappa}_{k} / \kappa_{D} \sigma_{k}\right)-\bar{\beta}_{2}\left(1-\gamma_{1}\right) \alpha_{k} \theta_{k} \bar{\kappa}_{k}^{2} \cdot \sqrt{1 / \sigma_{k} \bar{\kappa}_{k}}}{\varphi_{k}\left(s_{k}\right)} \\
& =\frac{\theta_{k} \alpha_{k} \bar{\kappa}_{k} \cdot\left[9 \bar{\beta}_{1} \max \left\{L_{g}+\kappa_{e g} \delta_{0}+\kappa_{H}, 1, \bar{\kappa}_{k}\right\}-\bar{\beta}_{2}\left(1-\gamma_{1}\right) \kappa_{D} \sqrt{\sigma_{k} \bar{\kappa}_{k}}\right]}{\kappa_{D} \sigma_{k} \varphi_{k}\left(s_{k}\right)} \\
& \quad \text { (37) } 0 .
\end{aligned}
$$

Thus, $\rho_{k} \geq \gamma_{1}$ and $\sigma_{k} \geq \sigma_{k+1}$ are from Step 7 .

Assumption 5. Let $\sigma_{k} \geq \sigma_{\min }>0$; then, $\sigma_{k} \longrightarrow \infty$ as $\alpha_{k} \longrightarrow 0$.

Assumption 5, which is reasonable when we use Armijo backing line search or Wolfe condition (ii) for global convergence, is important to promote global convergence.

If $y_{k}+\alpha_{k} d_{k}$ satisfies Armijo condition, $f\left(y_{k}+\alpha_{k} d_{k}\right)$ $\leq f\left(y_{k}\right)+c_{1} \alpha_{k} \nabla f\left(y_{k}\right)^{T} d_{k}$, where $c_{1}$ is in $(0,1)$, we assume that $\alpha_{k}=\alpha_{k, l}$, where $l$ is the first integer such that $\alpha_{k, l}$ satisfies Armijo condition, and we can get that 


$$
f\left(y_{k}+\frac{\alpha_{k}}{\lambda_{1}} d_{k}\right)>f\left(y_{k}\right)+\frac{\alpha_{k}}{\lambda_{1}} c_{1} \nabla f\left(y_{k}\right)^{T} d_{k} .
$$

Then, we can get that

$$
\begin{gathered}
\frac{\alpha_{k}}{\lambda_{1}}\left(\nabla f\left(y_{k}+\xi_{k} \frac{\alpha_{k}}{\lambda_{1}} d_{k}\right)-\nabla f\left(y_{k}\right)\right)^{T} d_{k}+\frac{\alpha_{k}}{\lambda_{1}}\left(1-c_{1}\right) \\
\cdot\left(\nabla f\left(y_{k}\right)-g_{k}\right)^{T} d_{k}+\frac{\alpha_{k}}{\lambda_{1}} c_{1} g_{k}^{T} d_{k}>0,
\end{gathered}
$$

where $\xi_{k} \in(0,1)$. From Assumption 2 and (5), then

$$
\left(\frac{\alpha_{k}}{\lambda_{1}}\right)^{2} L_{g}\left\|d_{k}\right\|^{2}+\frac{\alpha_{k}^{3}}{\lambda_{1}}\left(1-c_{1}\right) \kappa_{e g}\left\|d_{k}\right\|^{3}+\frac{\alpha_{k}}{\lambda_{1}} c_{1} g_{k}^{T} d_{k}>0 .
$$

Dividing (44) by $\left(\alpha_{k} / \lambda_{1}\right)$ and applying Lemma 2 ,

$$
\frac{\alpha_{k}}{\lambda_{1}} L_{g}\left\|d_{k}\right\|^{2}-\alpha_{k}^{2}\left(c_{1}-1\right) \kappa_{e g}\left\|d_{k}\right\|^{3}>c_{1} \bar{\beta}_{2} \bar{\kappa}_{k} \min \left\{1, \sqrt{\frac{\bar{\kappa}_{k}}{\sigma_{k}}}, \bar{\kappa}_{k}\right\} .
$$

If $\bar{\kappa}_{k}>\mathcal{E}$, using Assumption 3 and the fact that $\left\|d_{k}\right\|$ is bounded, $\left\|d_{k}\right\| \leq 3 \max \quad\left\{\left(\kappa_{H} / \sigma_{k}\right), \sqrt{\bar{\beta}_{1} \bar{\kappa}_{k} / \kappa_{D} \sigma_{k}}\right\} \leq 3$ $\max \left\{\left(\kappa_{H} / \sigma_{\min }\right), \sqrt{\kappa_{n g} / \sigma_{\min }}\right\}$, we can infer that $\sigma_{k} \longrightarrow \infty$ as $\alpha_{k} \longrightarrow 0$ from (45).

Then, we show that Assumption 5 can be also achieved when $y_{k}\left(\alpha_{k}\right)=y_{k}+\alpha_{k} d_{k}$ satisfies the Wolfe condition (ii):

$$
\nabla f\left(y_{k}+\alpha_{k} d_{k}\right)^{T} d_{k} \geq c_{2} \nabla f\left(y_{k}\right)^{T} d_{k}, \quad \text { in which } c_{2} \in(0,1) .
$$

We rewrite it as

$$
\begin{array}{r}
\left(\nabla f\left(y_{k}+\alpha_{k} d_{k}\right)-\nabla f\left(y_{k}\right)\right)^{T} d_{k}+\left(1-c_{2}\right) \\
\cdot\left(\nabla f\left(y_{k}\right)-g_{k}\right)^{T} d_{k}+\left(1-c_{2}\right) g_{k}^{T} d_{k}>0 .
\end{array}
$$

From Assumption 2 and (5) again, we have that

$$
L_{g} \alpha_{k}\left\|d_{k}\right\|^{2}+\left(1-c_{2}\right) \alpha_{k}^{2} \kappa_{e g} \kappa_{D}^{2}\left\|d_{k}\right\|^{3}+\left(1-c_{2}\right) g_{k}^{T} d_{k}>0 \text {. }
$$

Using Lemma 2 and Assumption 3, the following inequality,

$$
\begin{gathered}
L_{g} \alpha_{k}\left\|d_{k}\right\|^{2}+\left(1-c_{2}\right) \alpha_{k}^{2} \kappa_{e g} \kappa_{D}^{2}\left\|d_{k}\right\|^{3}>\left(1-c_{2}\right) \beta_{2} \bar{\kappa}_{k} \\
\cdot \min \left\{1, \sqrt{\frac{\bar{\kappa}_{k}}{\sigma_{k}}}, \bar{\kappa}_{k}\right\},
\end{gathered}
$$

holds. Consequently, $\lim _{\alpha_{k}} \rightarrow 0{ }_{k}=\infty$ if $\bar{\kappa}_{k}>\varepsilon$.

Assumption 6. Assume that $\operatorname{sign}\left([g(y)]_{j}\right)=\operatorname{sign}([\nabla f$ $\left.(y)]_{j}\right) \forall y \in B\left(y_{*}, \delta\right)$, when $\left[\nabla f\left(y_{*}\right)\right]_{j}=0, j=1,2, \ldots, n$.
Lemma 5. Let Assumptions 1-6 hold. If $|\mathscr{A}|<\infty$ and after many steps with a KKT points $y_{k}$ of (1), algorithm PFARC does not stop; then, $\lim _{k \rightarrow \infty} \bar{\kappa}_{k}=0$.

Proof. Choose an integer $K$ that satisfy that the filter is not augmented in iteration for all iteration $k \geq K$. Step 4 in algorithm PFARC gives that either (9) or (10) holds. If (9) holds for $k \geq K$, then $\left\|h\left(y_{k+1}\right)\right\| \leq\left(1-\eta_{h}\right)\left\|h\left(y_{k}\right)\right\| \leq \cdots \leq$ $\left(1-\eta_{h}\right)^{k-K}\left\|h\left(y_{K+1}\right)\right\|$ since $\eta_{h}$ is in $(0,1)$. Recall that $\bar{\beta}_{1} \bar{\kappa}_{k} \leq\left\|h\left(y_{k}\right)\right\|$, we infer that $\lim _{k \rightarrow \infty} \bar{\kappa}_{k}=0$ holds. If (9) does not hold for certain $k$ sufficiently large, algorithm DFFARC implies that, for each $k$ which is greater than $K$,

$$
f\left(y_{k+1}\right) \leq f\left(y_{k}\right)-\eta_{f} \alpha_{k}\left\|h\left(y_{k}\right)\right\|^{2} .
$$

Hence, for all $i=1,2, \ldots$,

$$
\begin{aligned}
f\left(y_{K+i}\right)= & \sum_{k=K}^{K+i-1}\left(f\left(y_{k+1}\right)-f\left(y_{k}\right)\right)+f\left(y_{K}\right) \leq \\
& -\sum_{k=K}^{K+i-1} \eta_{f} \alpha_{k}\left\|h\left(y_{k}\right)\right\|^{2}+f\left(y_{K}\right) .
\end{aligned}
$$

To have a contradiction, we let $\bar{\kappa}_{k}>\varepsilon$. Then, $\left\|h\left(y_{k}\right)\right\| \geq$ $\bar{\beta}_{1} \bar{\kappa}_{k} \geq \bar{\beta}_{1} \varepsilon$ and $f\left(y_{K}\right)-f\left(y_{K+i}\right) \geq \sum_{k=K}^{K+i-1} \eta_{f} \alpha_{k}\left\|h\left(y_{k}\right)\right\|^{2} \geq$ $\sum_{k=K}^{K} \eta_{f} \alpha_{k} \bar{\beta}_{1}^{2} \varepsilon^{2}$. Since $f\left(y_{K+i}\right)$ is bounded from below, the right-hand side series is bounded as $i \longrightarrow \infty$; then, $\lim _{k \longrightarrow 0} \alpha_{k}=0$.

Let $\alpha_{k}$ be given in Step 4 with $y_{k}+\alpha_{k} d_{k} \in \Omega$. It is easy to see that

$$
\forall y \in \Omega, D(y) \nabla f(y)=0 \Leftrightarrow[\nabla f(y)]_{j} \begin{cases}\geq 0, & \text { if }[y]_{j}=l_{j}, \\ \leq 0, & \text { if }[y]_{j}=u_{j}, \\ =0, & \text { if } l_{j} \leq[y]_{j} \leq u_{j},\end{cases}
$$

which is the KKT-conditions of (1). From the continuity of $f$, Assumption 6, and (5), we can get that $\left[g\left(y_{k}\right)\right]_{j}$ has the same sign when $y_{k}$ is close to $y$; then, $\alpha_{k} \rightarrow 0$ as $k \longrightarrow \infty$.

Furthermore, if $\alpha_{k}$ is obtained from the reduced conditions in Step 4, we have that $\sigma_{k} \longrightarrow \infty$ from Assumption 5. Recall that $\bar{\kappa}_{k}>\varepsilon$, we can have that, for sufficiently large $k$, $\sqrt{\sigma_{k} \bar{\kappa}_{k}}>\sqrt{\sigma_{k} \varepsilon}>\kappa_{H B}$; then, $\sigma_{k} \geq \sigma_{k+1}$ from Lemma 4 . According to the updating role in $\sigma_{k}$, if $\sigma_{k}<\sigma_{k+1}=\gamma^{-1} \sigma_{k}$, then $\sigma_{k} \leq\left(\kappa_{H B}^{2} / \varepsilon\right)<\left(\kappa_{H B}^{2} / \gamma \varepsilon\right)$ from Lemma 4. By induction, $\sigma_{0} \leq \max \left\{\sigma_{0},\left(\kappa_{H B}^{2} / \gamma \varepsilon\right)\right\}$. Suppose the claim that $\sigma_{k} \leq \max$ $\left\{\sigma_{0},\left(\kappa_{H B}^{2} / \gamma \varepsilon\right)\right\}$ holds. It is obvious that $\sigma_{k+1} \leq \max \left\{\sigma_{0},\left(\kappa_{H B}^{2}\right.\right.$ $\mid \gamma \varepsilon)\}$ if $\sigma_{k} \geq \sigma_{k+1}$. If $\sigma_{k}<\sigma_{k+1}$, then $\sigma_{k} \leq\left(\kappa_{H B}^{2} / \varepsilon\right)$; consequently, $\sigma_{k+1} \leq \max \left\{\sigma_{0},\left(\kappa_{H B}^{2} / \gamma \varepsilon\right)\right\}$ since $\sigma_{k+1}=\gamma^{-1} \sigma_{k}(\leq$ $\left.\left(\kappa_{H B}^{2} / \gamma \varepsilon\right)\right)$. The claim $\sigma_{k} \leq \sigma_{\max }=\max \left\{\sigma_{0},\left(\kappa_{H B}^{2} / \gamma \varepsilon\right)\right\}$, which contradicts to $\sigma_{k} \longrightarrow \infty$. Hence, $\alpha_{k} \nrightarrow 0$, and our hypothesis $\bar{\kappa}_{k}>\varepsilon$ is impossible. Thus, the conclusion holds.

Lemma 6. Let Assumptions 1-6 hold and $|\mathscr{A}|=\infty$. If algorithm PFARC does not stop after finite steps at a KKT points of $y_{k}$ in (1), then 


$$
\lim _{k \longrightarrow \infty, k \in \mathscr{A}} \bar{\kappa}_{k}=0
$$

Proof. To get a contradiction, we suppose that, for all large $k_{i}, \bar{\kappa}_{k_{i}} \geq \varepsilon$, where $\left\{k_{i}\right\}$ is a subsequence of $\mathscr{A}$ and $\varepsilon>0$ is a constant. By the definition of $k_{i}$, the pair of $\left(\left\|h\left(y_{k_{i}}\right)\right\|, f\left(y_{k_{i}}\right)\right)$ is augmented to $\mathscr{F}$. Thus, there exists an infinite subsequence $\left\{k_{i_{j}}\right\} \subseteq\left\{k_{i}\right\}$ such that $\bar{\kappa}_{k_{i_{j}}} \longrightarrow 0$ from the compactness theorem, which contradicts to $\bar{\kappa}_{k_{i}} \geq \varepsilon$. Hence, (53) holds.

Lemma 7. Let Assumptions 1-6 hold, and sequence $\left\{Q_{k}\right\}$ is probabilistically $\left(\kappa_{e h}, \kappa_{e q}, \kappa_{e f}\right)$ fully quadratic. $\left\{Y_{k}\right\}$ is a sequence of random iterates generated by algorithm PFARC. If any limit point $y_{*}$ of $\left\{y_{k}\right\}$ satisfies the strict complementarity, then liminf $\operatorname{lio}_{k \rightarrow \infty}\left\|D\left(Y_{k}\right) \nabla f\left(Y_{k}\right)\right\|=0$ almost surely.

Proof. Combining the affine scaling technique with the proof in Theorem 4.2 in [18], it is easy to derive the conclusion similar to Theorem 4.8 in [19]. We have omitted this section for reasons of length.

Theorem 1. Let Assumptions 1-5 hold, and algorithm PFARC does not stop after finite steps at a KKTpoints of $y_{k}$ in (1); thus,

$$
\lim _{k \longrightarrow \infty} \bar{\kappa}_{k}=0
$$

Proof. From Lemmas 5 and 6, we just think about this one case in which there are $k_{1}, k_{2}$ with $k_{1} \in \mathscr{A}$ and $k_{2} \notin \mathscr{A}$, and $k_{1}, k_{2}$ are larger than integer $K$. If (54) does not hold, there is a subsequence $\left\{y_{k_{i}}\right\}$ such that $\bar{\kappa}_{k_{i}} \geq 2 \varepsilon$ and $k_{i} \notin \mathscr{A}$. From Lemma 6, we also have, for every $k_{i}$, that $\bar{\kappa}_{l_{i}}<\varepsilon$; the iterate $y_{l_{i}}$ is the first iterate after $y_{k_{i}}$ such that $\left(\left\|h\left(y_{l_{i}}\right)\right\|, f\left(y_{l_{i}}\right)\right)$ is augmented to $\mathscr{F}$, that is, $l_{i} \in \mathscr{A}$. Thus,

$$
\bar{\kappa}_{k} \geq \varepsilon \text {, with } k_{i} \leq k<l_{i} \text { for all } i .
$$

Obviously, $\mathscr{K}=\left\{k \in \mathbb{N} \mid k_{i} \leq k<l_{i}\right\}$ is infinite, where $k_{i}$ and $l_{i}$ are defined as above.

For every $k=k_{i}, \ldots, l_{i}-1 \notin \mathscr{A}$, we have that either (9) or (10) holds. If (9) holds, then $\left(1-\eta_{h}\right)\left\|h\left(y_{k}\right)\right\| \geq\left\|h\left(y_{k+1}\right)\right\|$. Since $0<\eta_{h}<1$ and $\left\|h\left(y_{k}\right)\right\| \leq \kappa_{D} \kappa_{n g}$ and $\left\|h\left(y_{k+1}\right)\right\| \longrightarrow 0$, then $\bar{\kappa}_{k+1} \longrightarrow 0$ which contradicts to (55). Then, algorithm PFARC implies that (10) holds. Thus, $f\left(y_{k}\right)-$ $\eta_{f} \alpha_{k} \bar{\beta}_{1}^{2} \varepsilon^{2}>f\left(y_{k}\right)-\eta_{f} \alpha_{k}\left\|h\left(y_{k}\right)\right\|^{2} \geq f\left(y_{k+1}\right)$.

Moreover, $\left\{f\left(y_{k}\right)\right\}$ is monotonically decreasing and $f\left(y_{k_{i}+1}\right) \geq f\left(y_{l_{i}}\right)$. Consequently,

$$
\begin{gathered}
f\left(y_{k_{i}}\right)-\eta_{f} \alpha_{k_{i}} \bar{\beta}_{1}^{2} \varepsilon^{2}>f\left(y_{k_{i}}\right)-\eta_{f} \alpha_{k_{i}}\left\|h\left(y_{k_{i}}\right)\right\|^{2} \\
>f\left(y_{k_{i}+1}\right) \geq f\left(y_{l_{i}}\right), \quad \forall i .
\end{gathered}
$$

This ensures that $f\left(y_{k_{(i+1)}}\right) \geq f\left(y_{l_{i}}\right), \forall K \in \mathbb{N}$, with some $i \geq K$, because otherwise the inequality above will give that

$$
\begin{gathered}
f\left(y_{k_{i}}\right)-\eta_{f} \alpha_{k_{i}} \bar{\beta}_{1}^{2} \varepsilon^{2}>f\left(y_{k_{i}+1}\right) \leq f\left(y_{k_{i}}\right)-\eta_{f} \alpha_{k_{i}}\left\|h\left(y_{k_{i}}\right)\right\|^{2} \\
\geq f\left(y_{l_{i}}\right)>f\left(y_{k_{(i+1)}}\right), \quad \forall i .
\end{gathered}
$$

Similar to proof of Lemma 5, we can to prove that $\alpha_{k \in \mathscr{K}_{1}} \nrightarrow 0$, where $\mathscr{K}_{1} \subseteq \mathbb{N}$. Furthermore, from Assumption 5, we can get $\sigma_{k} \longrightarrow \infty$ as $\alpha_{k} \longrightarrow 0$. Recall that $\bar{\beta}_{1} \bar{\kappa}_{k} \leq \kappa_{D} \kappa_{n g}$ and $\left\|d_{k}\right\| \leq 3 \sqrt{\bar{\beta}_{1} \bar{\kappa}_{k} / \kappa_{D} \sigma_{k}}$; thus, $\left\|d_{k}\right\| \longrightarrow 0$, and then, $\theta_{k} \longrightarrow 1$, where $\theta_{k}$ is defined in Step 4 , so $s_{k}=\alpha_{k} d_{k}$. However,

$$
\begin{aligned}
-\bar{\varphi}_{k}\left(s_{k}\right) & =-\bar{\varphi}\left(\alpha_{k} d_{k}\right) \geq \alpha_{k} \bar{\beta}_{2} \bar{\kappa}_{k} \min \left\{1, \sqrt{\frac{\bar{\kappa}_{k}}{\sigma_{k}}}, \bar{\kappa}_{k}\right\} \\
\geq \alpha_{k} \bar{\beta}_{2} \varepsilon \cdot \frac{\kappa_{D}^{2}}{3 \bar{\beta}_{1}^{2}}\left\|d_{k}\right\| & =\bar{\beta}_{2} \varepsilon \cdot \frac{\kappa_{D}^{2}}{3 \bar{\beta}_{1}^{2}}\left\|s_{k}\right\|, \\
f\left(y_{k}+s_{k}\right)-f\left(y_{k}\right)-\varphi_{k}\left(s_{k}\right) & =\nabla f\left(y_{k}+\xi_{k} s_{k}\right)^{T} s_{k}-g_{k}^{T} s_{k}-\frac{1}{2} s_{k}^{T} H_{k} s_{k}-\frac{1}{3} \sigma_{k}\left\|s_{k}\right\|^{3} \\
& \leq\left[\nabla f\left(y_{k}+\xi_{k} d_{k}\right)-\nabla f\left(y_{k}\right)\right]^{T} s_{k}+\left[\nabla f\left(y_{k}\right)-g_{k}\right]^{T} s_{k}+\kappa_{H}\left\|s_{k}\right\|^{2} \\
\leq & \left(L_{g}+\kappa_{e g}\left\|s_{k}\right\|+\kappa_{H}\right)\left\|s_{k}\right\|^{2} .
\end{aligned}
$$


Thus, combining (58) and inequalities above, we can have for sufficiently large $k$ that

$$
\begin{gathered}
\rho_{k}=1-\frac{f\left(y_{k}+s_{k}\right)-f\left(y_{k}\right)-\varphi_{k}\left(s_{k}\right)}{-\varphi_{k}\left(s_{k}\right)} \geq 1 \\
-\frac{3 \bar{\beta}_{1}^{2}\left(L_{g}+\kappa_{e g}\left\|s_{k}\right\|+\kappa_{H}\right)\left\|s_{k}\right\|^{2}}{\bar{\beta}_{2} \kappa_{D}^{2} \varepsilon\left\|s_{k}\right\|} \geq \gamma_{2} .
\end{gathered}
$$

Then,

$$
\begin{aligned}
f\left(y_{k}\right)-f\left(y_{k+1}\right) & =f\left(y_{k}\right)-f\left(y_{k}+s_{k}\right) \\
& \geq \eta_{2}\left[-\varphi_{k}\left(s_{k}\right)\right] \geq \frac{\eta_{2} \bar{\beta}_{2} \kappa_{D}^{2} \varepsilon}{3 \bar{\beta}_{1}}\left\|s_{k}\right\| .
\end{aligned}
$$

Consequently,

$$
\left\|y_{k_{i}}-y_{l_{i}}\right\| \leq \sum_{k=k_{i}}^{l_{i}-1}\left\|s_{k}\right\| \leq \frac{3 \bar{\beta}_{1}}{\eta_{2} \bar{\beta}_{2} \kappa_{D}^{2} \varepsilon}\left[f\left(y_{k_{i}}\right)-f\left(y_{l_{i}}\right)\right] .
$$

Since $f\left(y_{k}\right)$ is convergent, we can get $\left\|y_{k_{i}}-y_{l_{i}}\right\| \longrightarrow 0$ from (61). However, $\bar{\kappa}$ is uniform continuous on $\mathscr{L}\left(x_{0}\right)$. Thus, $\bar{\kappa}_{k_{i}}-\bar{\kappa}_{l} \longrightarrow 0$ holds, which conflicts to the fact that $\left|\bar{\kappa}_{k_{i}}-\bar{\kappa}_{l_{i}}\right| \geq \varepsilon$. Therefore, our assumption is wrong and $\alpha_{k} \nrightarrow 0$.

Combining (57) with $\alpha_{k_{i}} \nrightarrow 0$, we get that $f\left(y_{k_{i}}\right) \longrightarrow-\infty$, which conflicts to $\left\{f\left(y_{k}\right)\right\}$ is bounded below. Thus, there is a subsequence $\left\{i_{j}\right\} \subset\{i\}$ such that

$$
f\left(y_{k_{\left(i_{j}+1\right)}}\right) \geq f\left(y_{i_{i_{j}}}\right) \text {. }
$$

Since $k_{\left(i_{j}+1\right)}$ is not in $\mathscr{A}$ and $\mathscr{F}_{l_{i j}} \subseteq \mathscr{F}_{k_{\left(i_{j}+1\right)}}$, it follows from (7) and (62) that

$$
\left(1-\eta_{h}\right)\left\|h\left(y_{l_{i_{j}}}\right)\right\| \geq\left\|h\left(y_{\left.k_{\left(i^{+1}\right.}\right)}\right)\right\| .
$$

For all $j, \quad l_{i_{j}} \in\left\{l_{i}\right\} \subseteq \mathscr{A}, \quad$ Lemma 6 implies $\lim _{j \rightarrow \infty}\left\|h\left(y_{l_{i j}}\right)\right\|=0$. Consequently, from (63), we get that $\lim _{j \longrightarrow \infty}\left\|h\left(y_{\underline{k}_{(i+1)}}\right)\right\|=0$; it contradicts to that $\left\|h\left(y_{k_{\left(i_{j+1}\right)}}\right)\right\| \geq \bar{\beta}_{1}^{\left(i_{j+1} \kappa_{k_{\left(i_{j+1}\right)}}\right.} \geq 2 \bar{\beta}_{1} \varepsilon$ since $k_{\left(i_{j+1}\right)} \notin \mathscr{A}$. Thus, the claim holds.

\section{Properties of the Local Convergence}

We can know that any limit point $y_{*}$ of (1) is a KKT point from Theorem 1 . In the following section, we consider the local convergence of algorithm PFARC, where the following assumptions are required.

Assumption 7. Assume that $\lim _{k \rightarrow \infty}\left\|\left(\nabla^{2} f\left(y_{k}\right)-H_{k}\right) d_{k}\right\| /$ $\left\|d_{k}\right\|=0$ as $\lim _{k \longrightarrow \infty} \bar{\kappa}_{k}=0$.

Assumption 8. Assume that $y_{*}$ satisfies

$$
\exists \kappa_{c}>0 \text { such that } d^{T} \nabla^{2} f\left(y_{*}\right) d \geq 2 \kappa_{c}\|d\|^{2}, \quad \forall d \neq 0 .
$$

Noting that $D\left(y_{*}\right)^{2} \nabla f\left(y_{*}\right)=0$ is equivalent to $D\left(y_{*}\right) \nabla f\left(y_{*}\right)=0$, then $\left\|D_{k}^{2} \nabla f\left(y_{k}\right)\right\| \longrightarrow 0$ is equivalent to the fact $\left\|D_{k} \nabla f\left(y_{k}\right)\right\| \longrightarrow 0$. Let $h_{1}(y)=\bar{D}(y)^{2} g(y)$. From (5), the fact $\left\|h_{1}\left(y_{k}\right)\right\| \longrightarrow 0$ is equivalent to the fact $\left\|h\left(y_{k}\right)\right\| \longrightarrow 0$, as $k \longrightarrow \infty$. To illustrate the properties of the local convergence, we substitute $h\left(y_{k}\right)$ for $h_{1}\left(y_{k}\right)$ in Step 4.4. That is, we accept the trial step $\alpha_{k, l}$ if

$$
\left\|h_{1}\left(y_{k}\left(\alpha_{k, l}\right)\right)\right\| \leq\left(1-\eta_{h}\right)\left\|h_{1}\left(y_{k}\right)\right\| \text { with } y_{k}+\alpha_{k, l} d_{k} \in \Omega
$$

holds in Step 4.4 in the following sections.

Theorem 2. Assume that Assumptions 1-8 hold and algorithm PFARC does not stop finite step at a KKT points $y_{k}$ of (1); then, $\left\|d_{k}\right\| \longrightarrow 0, \alpha_{k} \equiv 1$ for large $k$ and all iterations eventually satisfy $\rho_{k}>\gamma_{2}, \sigma_{k}$ is bounded from above as $k \longrightarrow \infty$.

Proof. Theorem 1 gives that $\lim _{k \longrightarrow 0} \bar{\kappa}_{k} \longrightarrow 0$ from the assumptions in Theorem 2. Consequently, Assumption 7 implies that $\lim _{k \rightarrow \infty}\left\|\left(\nabla^{2} f\left(y_{k}\right)-H_{k}\right) d_{k}\right\| /\left\|d_{k}\right\|=0$.

Thus, for large $k$,

$$
d_{k}^{T}\left(\nabla^{2} f\left(y_{k}\right)-H_{k}\right) d_{k} \leq \kappa_{c}\left\|d_{k}\right\|^{2}
$$

in which $\kappa_{c}$ appears in Assumption 8. And, we also have that $d_{k}^{T} \nabla^{2} f\left(y_{k}\right) d_{k} \geq 2 \kappa_{c}\left\|d_{k}\right\|^{2}$ for large $k$ from Assumptions 2 and 8. Then, we can infer that $2 \kappa_{c}\left\|d_{k}\right\|^{2} \leq d_{k}^{T}\left[\nabla^{2} f\left(y_{k}\right)\right.$ $\left.H_{k}\right] d_{k}+d_{k}^{T} H_{k} d_{k} \leq \kappa_{c}\left\|d_{k}\right\|^{2}+d_{k}^{T} H_{k} d_{k}$. As a consequence, $d_{k}^{T} H_{k} d_{k} \geq \kappa_{c}\left\|d_{k}\right\|^{2}$. So, $H_{k}$ is positive definite. And,

$$
\begin{gathered}
\kappa_{c}\left\|d_{k}\right\|^{2} \leq d_{k}^{T} H_{k} d_{k} \leq d_{k}^{T}\left[H_{k}+\sigma_{k}\left\|d_{k}\right\| I\right] d_{k} \\
\stackrel{(18)}{=}-g_{k}^{T} d_{k} \leq\left\|g_{k}\right\| \cdot\left\|d_{k}\right\| .
\end{gathered}
$$

Since $d_{k} \neq 0$, the first and last terms above give

$$
\left\|d_{k}\right\| \leq \frac{\left\|g_{k}\right\|}{\kappa_{c}}
$$

Otherwise, $-\varphi_{k}\left(d_{k}\right)=0$, from Lemma 2. This, however, contradicts $-\varphi_{k}\left(d_{k}\right)>0$ since $\bar{\chi}_{k} \neq 0$. Recall that

$$
\left\|\bar{D}_{k} g_{k}\right\|=0 \Leftrightarrow[g(y)]_{j} \begin{cases}\geq 0, & \text { if }[y]_{j}=l_{i}, \\ \leq 0, & \text { if }[y]_{j}=u_{i}, \\ =0, & \text { if } l_{j} \leq[y]_{j} \leq u_{j},\end{cases}
$$

and we can get $\left\|g_{k}\right\| \longrightarrow 0$, from $\bar{\kappa}_{k} \longrightarrow 0$. Thus, (68) gives that $\left\|d_{k}\right\| \longrightarrow 0$. Furthermore, $\theta_{k} \longrightarrow 1$, in which $\theta_{k}$ appears in Step 4. Then, $s_{k}=\alpha_{k} d_{k}$ and $\widehat{s}_{k}=\alpha_{k} \widehat{d}_{k}$ for large $k$.

Assumption 6 provides that $\operatorname{sign}\left[g_{k}\right]_{j}$ and $\operatorname{sign}\left[\nabla f\left(y_{k}\right)\right]_{j}$ is the same for large $k$ if $\nabla\left[f\left(y_{*}\right)\right]_{j}=0$. On the other side, (5) gives that $\operatorname{sign}\left[g_{k}\right]_{j}$ and $\operatorname{sign}\left[\nabla f\left(x_{k}\right)\right]_{j}$ are also the same for large $k$ if $\left[\nabla f\left(y_{*}\right)\right]_{j} \neq 0$ for some $j$. Thus, $D_{k}=\bar{D}_{k}$ for large $k$.

Now let us think about $\sigma_{k}$. From Step 7, $\rho_{k}=1-\left(f\left(y_{k}+s_{k}\right)-f\left(y_{k}\right)\left(-\varphi_{k}\left(s_{k}\right)\right)\right) /-\varphi_{k}\left(s_{k}\right)$.

However, using a Taylor expansion, for some $\xi_{k} \in(0,1)$, 


$$
\begin{aligned}
f\left(y_{k}+s_{k}\right) & -f\left(y_{k}\right)-\varphi_{k}\left(s_{k}\right) \\
& =\nabla f\left(y_{k}\right)^{T} s_{k}+\frac{1}{2} s_{k}^{T} \nabla^{2} f\left(y_{k}+\xi_{k} s_{k}\right) s_{k}-g_{k}^{T} s_{k}-\frac{1}{2} s_{k}^{T} H_{k} s_{k}-\frac{1}{3} \sigma_{k}\left\|s_{k}\right\|^{3} \\
& \leq\left(\nabla f\left(y_{k}\right)-g_{k}\right)^{T} s_{k}+\frac{1}{2} s_{k}^{T} \nabla^{2} f\left(y_{k}+\xi_{k} s_{k}\right) s_{k}-\frac{1}{2} s_{k}^{T} H_{k} s_{k} \\
& \leq \kappa_{D} \kappa_{e g} \bar{\kappa}_{k}\left\|s_{k}\right\|^{2}+\frac{1}{2} \kappa_{D}^{2}\left\|s_{k}\right\|^{2} \cdot\left\|\nabla^{2} f\left(y_{k}+\xi_{k} s_{k}\right)-\nabla^{2} f\left(x_{k}\right)\right\|+\frac{1}{2}\left\|s_{k}\right\| \frac{\left\|\left(\nabla^{2} f\left(y_{k}\right)-H_{k}\right) s_{k}\right\|,}{\left\|s_{k}\right\|}, \\
\varphi_{k}\left(s_{k}\right) & =g_{k}^{T} s_{k}+\frac{1}{2} s_{k}^{T} H_{k} s_{k}+\frac{1}{3} \sigma_{k}\left\|s_{k}\right\|^{3} \\
& \stackrel{(18)}{=}-\left[s_{k}^{T}\left(H_{k}+\sigma_{k}\left\|s_{k}\right\| I\right) s_{k}\right]+\frac{1}{2} s_{k}^{T} H_{k} s_{k}+\frac{1}{3} \sigma_{k}\left\|s_{k}\right\|^{3} \\
& =-\frac{1}{2} s_{k}^{T} H_{k} s_{k}-\frac{2}{3} \sigma_{k}\left\|s_{k}\right\|^{3} \leq-\frac{1}{2} s_{k}^{T} H_{k} s_{k} \leq-\frac{\kappa_{c}}{2}\left\|s_{k}\right\|^{2} .
\end{aligned}
$$

Together with the inequalities above, for large $k$,

$$
\rho_{k} \geq 1-\frac{\kappa_{D} \kappa_{e g} \bar{\kappa}_{k}+(1 / 2) \kappa_{D}^{2}\left\|\nabla^{2} f\left(y_{k}+\xi_{k} s_{k}\right)-\nabla^{2} f\left(y_{k}\right)\right\|+(1 / 2)\left(\left\|\nabla^{2} f\left(y_{k}\right) H_{k} s_{k}\right\| /\left\|s_{k}\right\|\right)}{\left(\kappa_{c} / 2\right)}>\gamma_{2},
$$

where the last inequality is from $\bar{\kappa}_{k} \longrightarrow 0$ and Assumptions 2 and 7. Recall the updating role in $\sigma_{k}$ in Step 7, and we get that

$$
\sigma_{k}>\sigma_{k+1}=\gamma \sigma_{k}, 0<\gamma<1, k \text { sufficiently large. }
$$

Thus, $\sigma_{k}$ is bounded from above and $\sigma_{k}\left\|d_{k}\right\| \longrightarrow 0$. Clearly, $\left\|h_{1}\left(y_{k}\right)\right\|=\left\|\bar{D}_{k}^{2} g_{k}\right\| \neq 0$. Set $\varepsilon_{k}=\left(1-\eta_{h}\right) /\left(2 \delta_{1}\right) \| h_{1}$ $\left(y_{k}\right) \|$, where $\delta_{1}$ is sufficiently small such that

$$
\begin{aligned}
\left\|h_{1}\left(y_{k}\right)+\nabla h_{1}\left(y_{k}\right) d_{k}\right\| & =\left\|\bar{D}_{k}^{2} g_{k}+\left(\bar{D}_{k}^{2} H_{k}+\bar{D}_{k} \operatorname{diag}\left\{g_{k}\right\} \bar{J}_{k}\right) d_{k}\right\| \\
& \stackrel{(18)}{\leq} \sigma_{k} \kappa_{D}^{2}\left\|d_{k}\right\|^{2}+\left\|\bar{D}_{k} g_{k}\right\| \cdot\left\|d_{k}\right\| \\
& \leq\left(\kappa_{D}^{2} \sigma_{k}\left\|d_{k}\right\|+\beta_{1} \bar{\kappa}_{k}\right)\left\|d_{k}\right\| \leq \varepsilon_{k}\left\|d_{k}\right\|,
\end{aligned}
$$

whenever $\left\|d_{k}\right\| \leq \delta_{1}$. Let $\omega_{k}=\left(\left(1-\eta_{h}\right) / 2 \delta_{2}\right)\left\|h_{1}\left(y_{k}\right)\right\|$, where $\delta_{2}$ is sufficiently small such that

$$
\left\|h_{1}\left(y_{k}+d_{k}\right)-h_{1}\left(y_{k}\right)-\nabla h_{1}\left(y_{k}\right) d_{k}\right\| \leq \omega_{k}\left\|d_{k}\right\|,
$$

whenever $\left\|d_{k}\right\| \leq \delta_{2}$. Such $\delta_{2}$ exists by Lemma 1.2 in [22].

Then, it follows that

$$
\begin{aligned}
\left\|\mid h_{1}\left(y_{k}+d_{k}\right)\right\| \leq & \left\|h_{1}\left(y_{k}+d_{k}\right)-h_{1}\left(y_{k}\right)-\nabla h_{1}\left(y_{k}\right) d_{k}\right\| \\
& +\left\|h_{1}\left(y_{k}\right)+\nabla h_{1}\left(y_{k}\right) d_{k}\right\| \\
\leq & \omega_{k}\left\|d_{k}\right\|+\varepsilon_{k}\left\|d_{k}\right\| \\
\leq & \frac{\left(1-\eta_{h}\right)}{\delta}\left\|h_{1}\left(y_{k}\right)\right\| \cdot\left\|d_{k}\right\| \\
\leq & \left(1-\eta_{h}\right)\left\|h_{1}\left(y_{k}\right)\right\|
\end{aligned}
$$

whenever $\left\|d_{k}\right\| \leq \delta$, where $\delta=\min \left\{\delta_{1}, \delta_{2}\right\}$.

Let $\alpha_{k}$ be given by $y_{k}+\alpha_{k} d_{k} \in \Omega$ in Step 4 . Similar to the proof of Lemma 5 , we can also get that $\alpha_{k} \nrightarrow 0$. Therefore, $\alpha_{k} \equiv 1$ for large $k$.

Corollary 1. Under the conditions of Theorem 2, then

$$
\begin{aligned}
& \frac{\left\|D_{k+1}^{2} \nabla f\left(y_{k+1}\right)\right\|}{\left\|D_{k}^{2} \nabla f\left(y_{k}\right)\right\|} \longrightarrow 0, \quad \text { as } k \longrightarrow \infty, \\
& \frac{\left\|y_{k+1}-y_{*}\right\|}{\left\|y_{k}-y_{*}\right\|} \longrightarrow 0, \quad \text { as } k \longrightarrow \infty .
\end{aligned}
$$

Proof. Recalling (5) and $D_{k}=\bar{D}_{k}$ for large $k$, we obtain the following inequalities:

$$
\begin{aligned}
\left\|D_{k+1} \nabla f\left(y_{k+1}\right)\right\| \leq & \left\|D_{k+1} \nabla f\left(y_{k+1}\right)-h\left(y_{k+1}\right)\right\| \\
& +\left\|h\left(y_{k+1}\right)\right\| \leq\left(\kappa_{D} \kappa_{e g} \bar{\kappa}_{k+1}+\bar{\beta}_{1}\right) \cdot \bar{\kappa}_{k+1}, \\
\left\|D_{k} \nabla f\left(y_{k}\right)\right\| \geq & \left\|h\left(y_{k}\right)\right\|-\left\|h\left(y_{k}\right)-D_{k} \nabla f\left(y_{k}\right)\right\| \\
\geq & \left(\bar{\beta}_{1}-\kappa_{D} \kappa_{e g} \bar{k}_{k}\right) \cdot \bar{\kappa}_{k} .
\end{aligned}
$$

Hence,

$$
\frac{\left\|D_{k+1} \nabla f\left(y_{k+1}\right)\right\|}{\left\|D_{k} \nabla f\left(y_{k}\right)\right\|} \leq \frac{\left(\kappa_{D} \kappa_{e g} \bar{\kappa}_{k+1}+\bar{\beta}_{1}\right) \cdot \bar{\kappa}_{k+1}}{\left(\bar{\beta}_{1}-\kappa_{D} \kappa_{e g} \bar{\kappa}_{k}\right) \cdot \bar{\kappa}_{k}} \longrightarrow 0,
$$

as $k \longrightarrow \infty$. The limit in (79) is from (75). In fact, (75) gives that $\left\|h_{1}\left(y_{k+1}\right)\right\|=o\left(\left\|h_{1}\left(y_{k}\right)\right\|\right)$ since $\left\|d_{k}\right\| \longrightarrow 0$. Thus, $\left\|h\left(y_{k+1}\right)\right\|=o\left(\left\|h\left(y_{k}\right)\right\|\right)$, Furthermore, $\bar{\kappa}_{k+1}=o\left(\bar{\kappa}_{k}\right)$. Noting 
that $\left\|D_{k}^{2} \nabla f\left(y_{k}\right)\right\| \longrightarrow 0$ is equivalently to $\left\|D_{k} \nabla f\left(y_{k}\right)\right\|$ $\longrightarrow 0$, we have that (76) holds.

Since $y_{*}$ is the limit point of $\left\{y_{k}\right\}$, there exist positive scales $\beta_{3}, \beta_{4}, \beta_{5}, \beta_{6}$, such that

$$
\begin{aligned}
& \beta_{3}\left\|y_{k+1}-y_{*}\right\| \leq\left\|D_{k+1}^{-2} \nabla f\left(y_{k+1}\right)\right\| \leq \beta_{4}\left\|y_{k}-y_{*}\right\|, \\
& \beta_{5}\left\|y_{k+1}-y_{*}\right\| \leq\left\|D_{k}^{-2} \nabla f\left(y_{k}\right)\right\| \leq \beta_{6}\left\|y_{k}-y_{*}\right\| .
\end{aligned}
$$

Consequently, (77) holds from the inequalities above and (76).

\section{Numerical Analysis}

The main task of this section is to test the algorithm PFARC constructed in this paper by solving actual test problems. We use MATLAB 2014a to program the algorithm, solve all the test problems in Table 1, and record the corresponding results. The initial parameters given by the algorithm PFARC are as follows:

$$
\begin{aligned}
\sigma_{0} & =1, \\
\delta_{0} & =0.5, \\
\gamma_{1} & =0.3, \\
\gamma_{2} & =0.7, \\
\gamma & =0.6, \\
\lambda_{1} & =0.1, \\
\lambda_{2} & =0.7, \\
\eta_{f} & =0.8, \\
\eta_{h} & =0.8, \\
\gamma_{3} & =0.5 .
\end{aligned}
$$

In this paper, the termination precision of PFARC is set to $\varepsilon=10^{-5}$.

In the following table, every test problem comes from [23], where $n$ represents the dimension of the problems and $l$ and $u$ represent the lower bound and upper bound of the problems, respectively.

Next, we will use PFARC and BOBYQA to solve the same problems in Table 1, respectively, and the results are recorded in Table 2, where $\mathrm{INTR}_{1}$ and $\mathrm{INTR}_{2}$ represent the number of iterations in PFARC and BOBYQA, respectively.

From the comparison results in Table 2, we can easily see that our algorithm PFARC can use a few iterations to get the optimal solution when solving the test problem given in Table 1, while BOBYQA algorithm needs a large number of iterations, which shows that PFARC has advantages in calculating the given test problems in Table 1.

In order to further test our algorithm, we will use PFARC given by this paper to calculate the problems given in [3], and the comparison results are recorded in Table 3. In Table 3, $n$ represents the dimension of the problems, $\mathrm{INTR}_{3}$ represents the number of iterations in PFARC, and $\mathrm{INTR}_{4}$ represents the number of iterations for the algorithm given in [3]. In addition, the questions in Table 3 are from [24].
TABle 1: The information of test problems.

\begin{tabular}{lccc}
\hline Problems & $n$ & $l$ & $u$ \\
\hline HS05 & 2 & {$[-1.5,-3]$} & {$[4,3]$} \\
HS18 & 2 & {$[2,0]$} & {$[50,50]$} \\
HS19 & 2 & {$[13,0]$} & {$[100,100]$} \\
HS21 & 2 & {$[2,-50]$} & {$[50,50]$} \\
HS23 & 3 & {$[-50,-50]$} & {$[50,50]$} \\
HS30 & 3 & {$[1,-10,-10]$} & {$[10,10,10]$} \\
HS31 & 3 & {$[-10,1,-10]$} & {$[10,10,1]$} \\
HS34 & 3 & {$[0,0,0]$} & {$[100,100,100]$} \\
HS36 & 3 & {$[0,0,0]$} & {$[20,11,42]$} \\
HS37 & 3 & {$[0,0,0]$} & {$[42,42,42]$} \\
HS38 & 4 & {$[-10,-10,-10,-10]$} & {$[10,10,10,10]$} \\
HS41 & 4 & {$[0,0,0,0]$} & {$[1,1,1,2]$} \\
HS45 & 5 & {$[0,0,0,0,0]$} & {$[1,2,3,4,5]$} \\
HS53 & 5 & {$[-10, \ldots,-10]$} & {$[10, \ldots, 10]$} \\
HS59 & 2 & {$[0,0]$} & {$[75,65]$} \\
HS60 & 3 & {$[-10,-10,-10]$} & {$[10,10,10]$} \\
HS62 & 3 & {$[0,0,0]$} & {$[1,1,1]$} \\
HS65 & 3 & {$[-5,-5,-5]$} & {$[5,5,5]$} \\
HS66 & 3 & {$[0,0,0]$} & {$[100,100,1]$} \\
HS68 & 4 & {$[0.0001,0,0,0]$} & {$[100,100,2,2]$} \\
HS71 & 4 & {$[1,1,1,1]$} & {$[5,5,5,5]$} \\
HS80 & 5 & {$[-2.3,-2.3,-3.2,-3.2$,} & {$[2.3,2.3,3.2,3.2,3.2]$} \\
HS83 & 5 & $-3.2]$ & {$[102,45,45,45,45]$} \\
HS110 & 10 & {$[2.001, \ldots, 23,27,27]$} & {$[9.999, \ldots, 9.999]$} \\
\hline & &
\end{tabular}

TABLE 2: The calculation results.

\begin{tabular}{lccc}
\hline Problems & $n$ & INTR $_{1}$ & INTR $_{2}$ \\
\hline HS05 & 2 & 4 & 145 \\
HS18 & 2 & 2 & 76 \\
HS21 & 2 & 22 & 467 \\
HS23 & 2 & 2 & 68 \\
HS25 & 3 & 2 & 40 \\
HS30 & 3 & 3 & 85 \\
HS31 & 3 & 3 & 99 \\
HS34 & 3 & 4 & 148 \\
HS36 & 3 & 4 & 171 \\
HS37 & 3 & 5 & 270 \\
HS38 & 4 & 17 & 385 \\
HS41 & 4 & 2 & 158 \\
HS45 & 5 & 2 & 189 \\
HS53 & 5 & 4 & 168 \\
HS59 & 2 & 10 & 387 \\
HS60 & 3 & 3 & 271 \\
HS62 & 3 & 3 & 233 \\
HS65 & 3 & 7 & 152 \\
HS66 & 3 & 6 & 311 \\
HS68 & 4 & 8 & 298 \\
HS71 & 4 & 3 & 73 \\
HS80 & 5 & 14 & 692 \\
HS83 & 5 & 14 & 771 \\
HS110 & 10 & &
\end{tabular}

From the results in Table 3 , it can be seen that the same problem is solved by the ARC method using the interpolation technology without derivative. The number of iterations required by PFARC is less than the number of iterations in [3]. The import reason is that filter technique 
TABLE 3: The calculation results.

\begin{tabular}{lccc}
\hline Problems & $n$ & INTR $_{3}$ & INTR $_{4}$ \\
\hline S201 & 2 & 2 & 4 \\
S203 & 2 & 5 & 7 \\
S206 & 2 & 3 & 5 \\
S207 & 2 & 6 & 7 \\
S212 & 3 & 5 & 9 \\
S213 & 3 & 12 & 27 \\
S240 & 3 & 1 & 3 \\
S256 & 4 & 10 & 16 \\
S261 & 4 & 12 & 12 \\
S283 & 10 & 10 & 34 \\
\hline
\end{tabular}

implemented in PFARC has two goals: minimization of $f$ and $\|h\|$, but a line search technique in [3] only performed the minimization of $f$. Therefore, our algorithm PFARC is more effective for solving bounded constrained derivativefree optimization problems.

In this paper, we propose a new algorithm PFARC for solving box constrained optimization problems without derivatives, which further complements the algorithm library for solving this kind of optimization. Since box constrained optimization is a classical problem, the proposed algorithm is an evolution of the existing algorithms, which further improved the existing ones. Box constrained optimization can be used in many fields, such as finance, science and technology, and military industry. Because the derivative information observed often is unavailable or unreliable, the derivative-free algorithm we proposed is closer to the reality. In the future, we can use the idea of this algorithm PFARC to solve other optimization problems, which is what we are going to do later.

\section{Data Availability}

The data used to support the findings of the study can be obtained from W. Hock, K. Schittkowski, Test examples for nonlinear programming codes, Lecture Notes in Economics and Mathematics System, 187. Springer (1981), and K. Schittkowski, MORE test examples for nonlinear programming codes, Lecture Notes in Economics and Mathematics System, 187. Springer (1981).

\section{Conflicts of Interest}

The authors declare that they have no conflicts of interest.

\section{Acknowledgments}

The authors gratefully acknowledge the partial supports of the Natural Science Foundation of Hainan Province (117107).

\section{References}

[1] N. Echebest, M. L. Schuverdt, and R. P. Vignau, "A derivativefree method for solving box-constrained underdetermined nonlinear systems of equations," Applied Mathematics and Computation, vol. 219, no. 6, pp. 3198-3208, 2012.
[2] U. M. García-Palomares, I. J. García-Urrea, and P. S. Rodríguez-Hernández, "On sequential and parallel nonmonotone derivative-free algorithms for box constrained optimization," Optimization Methods and Software, vol. 28, no. 6, pp. 1233-1261, 2013.

[3] X. Huang and D. Zhu, "An interior affine scaling cubic regularization algorithm for derivative-free optimization subject to bound constraints," Journal of Computational and Applied Mathematics, vol. 321, pp. 108-127, 2017.

[4] M. J. D. Powell, The BOBYQA Algorithm for Bound Constrained Optimization without Derivatives, Technical report, Department of Applied Mathematics and Theoretical Physics, University of Cambridge, London, UK, 2009.

[5] C. Cartis, N. I. M. Gould, and P. L. Toint, "Adaptive cubic regularisation methods for unconstrained optimization. Part I: motivation, convergence and numerical results," Mathematical Programming, vol. 127, no. 2, pp. 245-295, 2011.

[6] C. Cartis, N. I. M. Gould, and P. L. Toint, "Adaptive cubic regularisation methods for unconstrained optimization. Part II: worst-case function- and derivative-evaluation complexity," Mathematical Programming, vol. 130, no. 2, pp. 295-319, 2011.

[7] C. Cartis, N. I. M. Gould, and P. L. Toint, "On the oracle complexity of first-order and derivative-free algorithms for smooth nonconvex minimization," SIAM Journal on Optimization, vol. 22, no. 1, pp. 66-86, 2012.

[8] C. Cartis, N. I. M. Gould, and P. L. Toint, "An adaptive cubic regularization algorithm for nonconvex optimization with convex constraints and its function-evaluation complexity," IMA Journal of Numerical Analysis, vol. 32, no. 4, pp. 16621695, 2012.

[9] N. I. M. Gould, M. Porcelli, and P. L. Toint, "Updating the regularization parameter in the adaptive cubic regularization algorithm," Computational Optimization and Applications, vol. 53, no. 1, pp. 1-22, 2012.

[10] T. F. Coleman and Y. Li, "An interior trust region approach for nonlinear minimization subject to bounds," SIAM Journal on Optimization, vol. 6, no. 2, pp. 418-445, 1996.

[11] D. Zhu, "An affine scaling trust-region algorithm with interior backtracking technique for solving bound-constrained nonlinear systems," Journal of Computational and Applied Mathematics, vol. 184, no. 2, pp. 343-361, 2005.

[12] G. Fasano, J. L. Morales, and J. Nocedal, "On the geometry phase in model-based algorithms for derivative-free optimization," Optimization Methods and Software, vol. 24, no. 1, pp. 145-154, 2009.

[13] S. Gratton, P. L. Toint, and A. Troltzsch, "An active-set trustregion method for derivative-free nonlinear bound-constrained optimization," Optimization Methods and Software, vol. 26, no. 4-5, pp. 873-894, 2011.

[14] M. J. D. Powell, The NEWUOA Software for Unconstrained Optimization without Derivatives, Technical Report DAMTP NA2004/08, Department of Applied Mathematics and Theoretical Physics, Cambridge University, Cambridge CB3 9EW, London, UK, 2004.

[15] M. J. D. Powell, "Developments of NEWUOA for minimization without derivatives," IMA Journal of Numerical Analysis, vol. 28, no. 4, pp. 649-664, 2008.

[16] A. R. Conn, K. Scheinberg, and L. N. Vicente, "Global convergence of general derivative-free trust-region algorithms to first- and second-order critical points," SIAM Journal on Optimization, vol. 20, no. 1, pp. 387-415, 2009.

[17] D. Li and D. Zhu, "An affine scaling interior trust-region method combining with line search filter technique for 
optimization subject to bounds on variables," Numerical Algorithms, vol. 77, no. 4, pp. 1159-1182, 2018.

[18] A. S. Bandeira, K. Scheinberg, and L. N. Vicente, "Convergence of trust-region methods based on probabilistic models," SIAM Journal on Optimization, vol. 24, no. 3, pp. 1238-1264, 2014.

[19] P. Wang and D. Zhu, "A derivative-free affine scaling trust region methods based on probabilistic models with new nonmonotone line search technique for linear inequality constrained minimization without strict complementarity," International Journal of Computer Mathematics, vol. 96, no. 4, pp. 663-691, 2018.

[20] A. R. Conn, K. Scheinberg, and L. N. Vicente, Introduction to Derivative-free Optimization, SIAM, Philadelphia, PA, USA, 2009.

[21] M. Ulbrich, "Nonmonotone trust-region methods for boundconstrained semismooth equations with applications to nonlinear mixed complementarity problems," SIAM Journal on Optimization, vol. 11, no. 4, pp. 889-917, 2001.

[22] S. C. Eisenstat and H. F. Walker, "Globally convergent inexact Newton methods," SIAM Journal on Optimization, vol. 4, no. 2, pp. 393-422, 1994.

[23] W. Hock and K. Schittkowski, Test Examples for Nonlinear Programming Codes, Springer, Berlin, Germany, Lecture Notes in Economics and Mathematics System, 1981.

[24] K. Schittkowski, MORE Test Examples for Nonlinear Programming Codes, Springer, Berlin, Germany, Lecture Notes in Economics and Mathematics System, 1981. 